\title{
Leave to appeal
}

\author{
We can publish only a small fraction of the papers that are submitted to Nature Physics. If you think \\ we've got it wrong in declining your work, what should you do about it?
}

No one who submits their work to Nature Physics does so unless they believe it to be groundbreaking and therefore deserving of a place in the journal. We are grateful for the consistently high quality of manuscripts we receive; regrettably, however, we can only publish around $10 \%$ of them. We never make a decision lightly, but try to ensure that we have understood the contribution and context of each and every paper that is submitted. If we've declined your paper, we've done so for a reason.

But we don't always get it right. So we are always prepared to reconsider any decision. If you feel that we have misjudged your work, send an e-mail (to naturephysics@nature.com) explaining the key reasons why you feel that your work has been misjudged - but before you do, there are a number of things to consider.

We can reverse a decision only if it becomes clear that we, or our referees, have overlooked or misconstrued specific scientific issues related to the work in question, or if implications are pointed out that were not already apparent from the manuscript itself. And we will do so only if the grounds for appeal are compelling: in practice, this is true only in a minority of cases.
The overwhelming majority of submissions - more than $70 \%$ - are declined without external review. Although deciding whether or not a paper deserves to be published in Nature Physics is never easy, such decisions are the prerogative of its editors. The argument that experts are likely to be impressed by a work is not sufficient grounds for appeal.

For those papers that are sent to review, we do not ask referees to say whether or not they think that a paper belongs in Nature Physics. Instead, we ask them to tell us specifically what they feel to be the paper's contribution, and what its significance might be. Based on these comments and our own editorial criteria we decide whether publication in Nature Physics is appropriate. Certainly, we do not base our decisions on a simple 'show of hands'.

Similarly, a paper should at the very least excite the experts in the field. If a paper elicits only a lukewarm response from our referees we are unlikely to be persuaded that it belongs in Nature Physics, rather than in a more topical or specialist journal, even if their comments were otherwise generally positive.

Comparisons to previous papers that have appeared in Nature Physics or elsewhere are rarely helpful. Of course we make every effort to be consistent in the decisions we make. But different works inevitably have different strengths and weaknesses, and each submission must be assessed on its own merits. Moreover, the editorial criteria we apply to the papers in any given field will inevitably change as the field progresses. We must also find the right balance between the papers we publish on 'hot topics' and those from the many other areas that are the remit of this journal.

The likelihood that a submission will be highly cited is also not a sufficient reason for it to be published in Nature Physics. We are, of course, pleased when the papers we publish are cited. However, many of the papers that we are proudest to have published are not the most cited; a high number of rapid citations is no guarantee of a paper's long-term impact or fundamental importance.

Nature Physics aims to publish the most important advances in fundamental physics and technological potential. We strive to ensure that every decision we make is based on a thorough understanding of the contribution and context of the papers we receive. We do ask our authors to consider whether there truly are compelling grounds to appeal against the decision we have made, but when it is clear we have made a mistake we appreciate the opportunity to correct it. $\square$

\section{Where credit is due}

\section{The 2010 Wolf Prize in Physics acknowledges research into the foundations of quantum mechanics.}

Since 1978, the Wolf Prizes have been awarded in mathematics, physics, chemistry, agriculture, medicine and arts, "to outstanding scientists and artists - irrespective of nationality, race, colour, religion, sex or political views for achievements in the interest of mankind and friendly relations among peoples". The 2010 winners in physics are John Clauser, Alain Aspect and Anton Zeilinger. It brings a purse of $\$ 100,000$, but also the prestige of one of the most important science prizes after the Nobel Prize - indeed, it can be an indicator for the latter: 14 out of the previous 45 recipients of the Wolf Prize in Physics went on to Nobel laureateship.
Clauser, Aspect and Zeilinger were cited at last month's ceremony for "their fundamental conceptual and experimental contributions to the foundations of quantum physics, specifically an increasingly sophisticated series of tests of Bell's inequalities or extensions thereof using entangled quantum states". Their work has established a good part of what we now call quantum information science. But to see it as a 'quantum information prize' would be to miss the point. Rather, it acknowledges the roots of that nowthriving field in what was, for a long time, a shadowy corner of physics.

When Clauser, Aspect and Zeilinger set to work, it was unclear whether the foundational basis for quantum mechanics hadn't been long since laid. But these three, building on work by John Bell, showed that important foundational issues remained. Their progress, against the prevailing attitude of the time, didn't come without sacrifice. John Clauser - one of the first to realize the full implications of Bell's work and to conduct experiments in the field never gained a permanent position in physics, and left the field. At last his seminal work is recognized, and his inclusion in this award is to be applauded.

There is always a risk that awards and recognition may distort the historical record. But they may also serve to set it straight. 